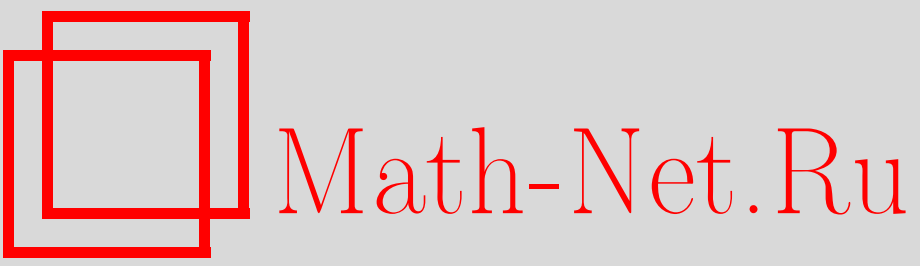

М. М. Чобан, О некоторых вопросах дескриптивной теории множеств в топологических пространствах, УМН, 2005, том 60, выпуск 4, 123-144

DOI: https://doi.org/10.4213/rm1447

Использование Общероссийского математического портала Math-Net.Ru подразумевает, что вы прочитали и согласны с пользовательским соглашением

http://www.mathnet.ru/rus/agreement

Параметры загрузки:

IP : 35.173 .137 .237

26 апреля 2023 г., 15:41:42 


\title{
О НЕКОТОРЫХ ВОПРОСАХ ДЕСКРИПТИВНОЙ ТЕОРИИ МНОЖЕСТВ В ТОПОЛОГИЧЕСКИХ ПРОСТРАНСТВАХ
}

\author{
М. М. ЧОБАН
}

\begin{abstract}
Вопросы о структуре борелевских множеств, об их классификации и о сохранении определенных свойств множеств при заданных типах отображений возникли в первой половине прошлого столетия в работах А. Лебега, Р. Бэра, Н.Н. Лузина, П. С. Александрова, П. С. Урысона, П. С. Новикова, Л. В. Келдыш, А. А. Ляпунова и породили многие исследования. В данной статье получены некоторые результаты, связанные с вопросами $\Phi$. Хаусдорфа, Н. Н. Лузина, П. С. Александрова, П. С. Урысона, М. Катетова, А. Стоуна.

В 1934 г. Хаусдорфом был поставлен вопрос о сохранении свойства быть абсолютным $B$-множеством (т.е. борелевским множеством некоторого полного сепарабельного метрического пространства) при открытых непрерьвных отображениях. В силу одной теоремы Л. В. Келдыш в общем случае ответ на этот вопрос отрицателен. В настоящей работе приведены дополнительные условия, при которых ответ на вопрос Хаусдорфа положителен.

В работе рассматриваются и некоторые общиевопросы теории операций над множествами.

Библиография: 56 названий.
\end{abstract}

\section{СОДЕРЖАНИЕ}

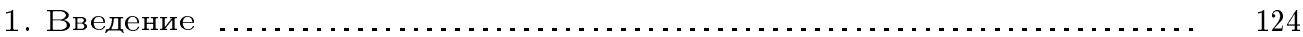

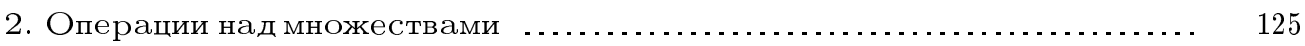

3. Бэровские пространства ...................................... 127

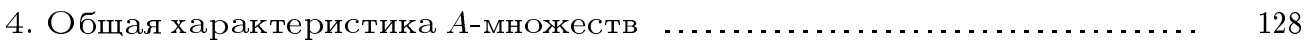

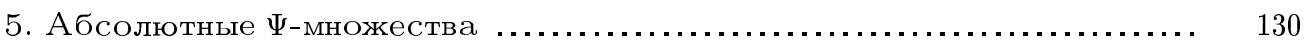

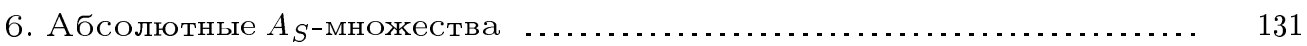

7. Ядра отображений ............................................. 132

8. Проблема Хаусдорфа об открытых отображениях. Общий случай .. 133

9. Проблема Хаусдорфа. Случай равномерно полных отображений ... 135

10. Проблема Хаусдорфа. Случай счетнократных отображений ....... 137

11. Проблема Хаусдорфа. Случай изолированных отображений $\ldots . . . .138$

12. О строении подмножеств в некоторых пространствах ................ 139

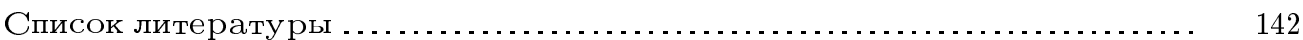

(C) М. М. Чован 2005 


\section{1. Введение}

Одним из важных направлений дескриптивной теории множеств является изучение зависимости между топологическим строением множеств и операциями, при помощи которых они построены, отправляясь от множеств более простой природы.

Дескриптивная теория множеств и функций родилась в трудах знаменитых французских математиков Э. Бореля, А. Лебега и Р. Бэра на рубеже 19 и 20 столетий (см. [5], [23], [32], [37], [38], [42], [44], [55], [6], [7], [17], [19], [34], [35], [36], [49]. Общая теория операций над множествами была построена в работах П. С. Александрова, Ф. Хаусдорфа, А.Н. Колмогорова, Л.В. Канторовича и Е.М. Ливенсона (см. [5], [38], [42], [44], [13], [49]). В работах Н. Н. Лузина, П. С. Александрова, Ф. Хаусдорфа, В. Серпинского, К. Куратовского, М. Суслина, Ш.-Ж. Валле-Пуссена, А.Н. Колмогорова, Л. В. Канторовича, Е. М. Ливенсона, П. С. Новикова, Л. В. Келдьш, А. Д. Тайманова, А. А. Ллпунова была разработана структурная теория $B$-множеств, благодаря которой классическая дескриптивная теория множеств приняла в целом законченньй вид.

Начиная с 30-х годов минувшего столетия дескриптивная теория множеств стала развиваться в следующих направлениях.

1. Теория операций над множествами.

2. Аксиоматическая дескриптивная теория множеств.

3. Дескриптивная теория множеств в обших пространствах.

4. Приложения дескриптивной теории множеств.

Отдельные достижения дескриптивной теории множеств в этих направлениях отражены в работах [5], [23], [37], [38], [42], [44], [55], [13], [7], [19], [21], [34], [49].

Основное содержание настояшей работы связано с решением Л.В. Келдыш [33] проблемы $\Phi$. Хаусдорфа [20] о сохранении классов борелевских множеств при открытых непрерьвных отображениях. Нами не только усилен этот результат, но и указаны некоторые ограничения, при которых проблема Хаусдорфа имеет положительное решение.

В работе рассматриваются и другие вопросы, связанные с решением проблем Н. Н. Лузина, П. С. Александрова, П. С. Урысона, А. Стоуна, М. Катетова, З. Фролика.

В работе используются следуюшие обозначения.

1. Через $\mathrm{cl}_{X} A$ или сl $A$ обозначается замькание множества $A$ в пространстве $X$.

2. Мощность множества $A$ обозначается через $|A|$.

3. На множестве $N=\{1,2, \ldots\}$ рассматривается только дискретная топология. Через $\mathbb{R}$ обозначается пространство действительных чисел, $I=[0,1] \subseteq \mathbb{R}$.

4. Через $w(X)$ обозначается вес пространства $X$.

5 . Если $\tau$ является кардинальньм числом, то через $\tau^{+}$обозначается первый кардинал больше $\tau$.

Кардинальное число $\tau$ отождествляется с первым ординалом мощности $\tau$.

Пусть $\omega$ есть первое бесконечное ординальное число, а $\omega_{1}$ есть первое несчетное ординальное число.

6. Применяется терминология из [13], [15], [20], [34]. Каждое пространство предполагается вполне регулярньм $T_{2}$-пространством. 


\section{2. Операции над множествами}

Для всякого кардинального числа $\tau$ через $J_{\tau}$ обозначим некоторое множество мощности $\tau$, вполне упорядоченное по всем порядковьм числам мощности меньше $\tau$. Кардинальное число $\tau$ отождествляется с первым порядковым числом мошности $\tau$.

Множество $J_{\tau}$ назьвается пространством индексов.

2.1. ОПРЕДЕЛЕниЕ. $\tau$-операцией назьвается всякая функция $\Psi$, ставящая в соответствие каждой таблице $\left\{E_{\alpha}: \alpha \in J_{\tau}\right\}$ подмножеств заданного множества $Z$ некоторое подмножество $E \subseteq Z$. В этом случае пишем $\Psi\left\{E_{\alpha}\right\}=E$, а $E$ назьвается результатом операции $\Psi$ над таблицей $\left\{E_{\alpha}\right\}$ относительно множества $Z$.

Две $\tau$-операции $\Psi, \Phi$ равны, если $\Psi\left\{E_{\alpha}\right\}=\Phi\left\{E_{\alpha}\right\}$ для любой таблицы подмножеств $\left\{E_{\alpha}\right\}$ произвольного множества $Z$.

Для любого семейства $\mathscr{L}$ подмножеств множества $Z$ и $\tau$-операции $\Psi$ через $\Psi(\mathscr{L})$ обозначим совокупность всех результатов операции $\Psi$ над таблищами вида $\left\{E_{\alpha}: \alpha \in J_{\tau}\right\} \subseteq \mathscr{L}$. Две операции $\Psi$ и $\Phi$ эквивалентны (обозначение: $\Psi \sim \Phi$ ), если $\Psi(\mathscr{L})=\Phi(\mathscr{L})$ для любого семейства подмножеств $\mathscr{L}$.

Равные операции эквивалентны. Мы будем отождествлять эквивалентные операции.

2.2. ОпРЕДЕлЕния. $\tau$-операция $\Psi$ называется:

а) положительной, если из $H_{\alpha} \subseteq E_{\alpha}$ для всех $\alpha \in J_{\tau}$ вытекает $\Psi\left\{H_{\alpha}\right\} \subseteq \Psi\left\{E_{\alpha}\right\}$;

б) экстенсивной, если $\mathscr{L} \subseteq \Psi(\mathscr{L})$ для всякого семейства подмножеств $\mathscr{L}$;

в) нормальной, если $\mathscr{L} \subseteq \Psi(\Psi(\mathscr{L})) \subseteq \Psi(\mathscr{L})$ для всякого семейства подмножеств $\mathscr{L}$.

Операция $\Psi$ сильнее операции $\Phi$ (обозначение: $\Phi \leqslant \Psi$ ), если $\Phi(\mathscr{L}) \subseteq \Psi(\mathscr{L})$ для всякого семейства подмножеств $\mathscr{L}$.

Всякое подмножество множества $J_{\tau}$ называется $\tau$-цепю. Любое множество $\tau$-цепей будем назьвать $\tau$-базой.

C $\tau$-базой $S$ можно связать следуюшие операции над множествами:

$$
\begin{aligned}
& \Psi_{S}\left\{E_{\alpha}\right\}=\bigcup\left\{\left(\bigcap\left\{E_{\alpha}: \alpha \in \xi\right\}\right) \cap\left(\bigcap\left\{Z \backslash E_{\beta}: \beta \notin \xi\right\}\right): \xi \in S\right\}, \\
& \Phi_{S}\left\{E_{\alpha}\right\}=\bigcup\left\{\bigcap\left\{E_{\alpha}: \alpha \in \xi\right\}: \xi \in S\right\} .
\end{aligned}
$$

При этом если $\xi=\varnothing$, то $\bigcap\left\{E_{\alpha}: \alpha \in \xi\right\}=Z$ для любой таблицы $\left\{E_{\alpha}\right\}$ подмножеств множества $Z$. ей.

Операция $\Psi_{S}$ называется $\tau$-st-операцией, а операция $\Phi_{S}$ назьвается $\tau$ - $\delta s$-операци-

Положительная $\tau$-st-операция эквивалентна $\tau$ - $\delta s$-операции.

Если $m<\tau$, то всякая $m$-st-операция эквивалентна $\tau$-st-операции.

База $S$ назьвается полной, если из $\xi \subseteq \eta$ и $\xi \in S$ следует $\eta \in S$. Если база $S$ полна, то $\Phi_{S}=\Psi_{S}$. Поэтому $\tau$ - $\delta s$-операция равна $\tau$-st-операции.

Для каждой $\tau$-операции $\Psi$ можно построить дополнительную $\tau$-операцию $\Psi^{c}$, где $\Psi^{c}\left\{E_{\alpha}\right\}=Z \backslash \Psi\left\{Z \backslash E_{\alpha}\right\}$. Если $S$ является $\tau$-базой и $S^{c}=\left\{\mu \in J_{\tau}: J_{\tau} \backslash \mu \notin S\right\}$, то $\left(\Psi_{S}\right)^{c}=\Psi_{S^{c} \text { и }}\left(\Phi_{S}\right)^{c}=\Phi_{S^{c}}$. 
2.3. ПримеРЫ. 1. Пусть $m \leqslant \tau$. Обозначим через $P^{m}$ операцию пересечения не более $m$ множеств. База $\tau$ - $\delta s$-операции $P^{m}$ состоит из одной цепи мошности $m$.

2 . Пусть $m \leqslant \tau$. Обозначим через $S^{m}$ операцию объединения не более $m$ множеств. База $\tau$ - $\delta s$-операции $S^{m}$ состоит из $m$ одноэлементных цепей.

3. Пусть $T_{\beta}=\Phi_{\{\beta\}}$. Тогда $T_{\beta}\left\{E_{\alpha}\right\}=E_{\beta}$ и $T_{\beta}$ называется тривиальной $\tau$-операцией. Все тривиальные операции эквивалентны.

4. Через $C_{\beta}$ обозначим $\tau$-st-операцию с базой $\left\{\xi \subseteq J_{\tau}: \beta \notin \xi\right\}$. Имеем $C_{\beta}\left\{E_{\alpha}\right\}=$ $Z \backslash E_{\beta}$. Операция $C_{\beta}$ назьвается операцией взятия дополнения.

5. Положим $\Psi\left\{E_{\alpha}\right\}=\bigcap\left\{E_{\alpha}: \alpha \in J_{\tau}\right\}$, если хотя бы одно из множеств $E_{\alpha}$ конечно, и $\Psi\left\{E_{\alpha}\right\}=\bigcup\left\{E_{\alpha}: \alpha \in J_{\tau}\right\}$, если все множества $E_{\alpha}$ бесконечны. Операция $\Psi$ экстенсивна, положительна и нормальна, но не является $\tau$-st-операцией.

Операции $P^{m}$ и $S^{m}$ взаимно дополнительны при соответствующем выборе баз.

Пусть $\tau^{+}$есть первое бесконечное кардинальное число, следующее за $\tau$.

С $\tau$-операцией $\Psi$ и семейством подмножеств $\mathscr{L}$ множества $Z$ можно связать трансфинитную последовательность семейств $\left\{\Psi_{\alpha}(\mathscr{L}): \alpha<\tau^{+}\right\}$, где $\Psi_{0}(\mathscr{L})=\mathscr{L}$, $\Psi_{\alpha}(\mathscr{L})=\Psi\left(\bigcup\left\{\Psi_{\beta}(\mathscr{L}): \beta<\alpha\right\}\right)$, если $\alpha$ четно, и $\Psi_{\alpha}(\mathscr{L})=\Psi^{c}\left(\bigcap\left\{\Psi_{\beta}(\mathscr{L}): \beta<\alpha\right\}\right)$, если $\alpha$ нечетно.

Пусть $X$ - топологическое пространство. Обозначим через $\mathscr{F}(X)$ совокупность всех замкнутых подмножеств пространства $X$, через $\mathscr{U}(X)$ - совокупность всех открытых подмножеств пространства $X, L(X)=\{F \cap U: F \in \mathscr{F}(X), U \in \mathscr{U}(X)\}$ и $Z(X)=\left\{f^{-1}(0): f: X \rightarrow \mathbb{R}\right.$ есть непрерывная функция $\}$. Множества из $L(X)$ называются $L$-множествами или локально замкнутыми множествами пространства $X$. Множества из $Z(X)$ назьваются нуль-множествами пространства $X$.

Пересечение счетного семейства $L$-множеств называется $L_{\delta}$-множеством.

$\tau$-операция $\Psi$ порождает на пространстве $X$ следуюшие семейства:

$$
\begin{gathered}
\Psi_{\alpha}(X)=\Psi_{\alpha}(\mathscr{F}(X)), \quad \Psi_{\alpha}(X, \mathscr{U})=\left\{X \backslash H: H \in \Psi_{\alpha}(X)\right\}=\Psi_{\alpha}^{c}(\mathscr{U}(X)), \\
\Psi_{\alpha}(X, L)=\Psi_{\alpha}(L(X)), \quad \Psi_{\alpha}(X, Z)=\Psi_{\alpha}(Z(X)),
\end{gathered}
$$

где $0 \leqslant \alpha<\tau^{+}$

Положим

$$
\begin{aligned}
& r(\Psi, X)=\bigcup\left\{\Psi_{\alpha}(X): \alpha<\tau^{+}\right\}, \\
& a(\Psi, X)=\bigcup\left\{\Psi_{\alpha}(X, L): \alpha<\tau^{+}\right\}, \\
& b(\Psi, X)=\bigcup\left\{\Psi_{\alpha}(X, Z): \alpha<\tau^{+}\right\} .
\end{aligned}
$$

Тогда $r(\Psi, X)$ называется $\Psi$-кольцом подмножеств пространства $X, a(\Psi, X)$ называется $\Psi$-алгеброй подмножеств пространства $X$.

Если $\tau=\aleph_{0}$, то $\delta=P^{\tau}, \sigma=S^{\tau}, \mathscr{F}_{\alpha}(X)=\sigma_{\alpha}(X), \mathscr{U}_{\alpha}(X)=\delta_{\alpha}(X, \mathscr{U}), B_{\alpha}(X)=$ $\sigma_{\alpha}(X, L), B a_{\alpha}(X)=\sigma_{\alpha}(X, Z), B o(X)=a(\delta, X)=a(\sigma, X)$ есть $\sigma$-алгебра борелевских множеств, $B o^{*}(X)=r(\delta, X)=r(\sigma, X)=\bigcup\left\{\mathscr{F}_{\alpha}(X): \alpha<\omega_{1}\right\}$ есть кольцо борелевских множеств и $B a(X)=b(\delta, X)=b(\sigma, X)$ есть $\sigma$-алгебра бэровских множеств пространства $X$.

Всегда $B a(X) \subseteq B o^{*}(X) \subseteq B o(X)$.

Следующие включения равносильны:

1) $B o^{*}(X) \subseteq B a(X)$;

2) $B o(X) \subseteq B a(X)$;

3) $B a(X)=B o^{*}(X)=B o(X)$. 
Если пространство $X$ совершенно нормально, то $B a(X)=B o(X)$.

2.4. Вопрос (М. Катетов, см. [18]). Является ли совершенно нормальным нормальное пространство $X$, для которого $B a(X)=B o(X)$ ?

Ответ на вопрос М. Катетова положителен, если пространство $X$ допускает такое непрерьвное отображение $g: X \rightarrow Y$ на некоторое метрическое пространство, что все прообразы $g^{-1}(y)$ псевдокомпактны и множество $g(H)$ замкнуто в $Y$, как только $H \in Z(X)$. В общем случае ответ неизвестен.

\section{3. Бэровские пространства}

Рассмотрим кардинальные числа $\tau$ и $m$. Предположим, что число $m$ бесконечно. На множестве $J_{\tau}$ рассмотрим дискретную топологию.

Для каждого $\alpha \in J_{m}$ положим $J_{\tau \alpha}=J_{\tau}$. Рассмотрим множество $B(\tau, m)=$ $\prod\left\{J_{\tau \alpha}: \alpha \in J_{m}\right\}$. Если $\xi=\left(\xi_{\alpha}: \alpha \in J_{m}\right) \in B(\tau, m)$ и $\beta \in J_{m}$, то $\xi \mid \beta=\left(\xi_{\alpha}: \alpha<\beta\right)$ является кортежем длины $\beta$. Для каждого кортежа $k=\left(\xi_{\alpha}: \alpha<\beta\right)$ длины $\beta<m$ положим $\delta(k)=\{\eta \in B(\tau, m): \eta \mid \beta=k\}$. Множества вида $\delta(k)$ образуют базу пространства $B(\tau, m)$ и называются бэровскими интервалами. Пространство $B(\tau, m)$ называется бэровским пространством ширины $\tau$, глубины $m$ и веса $\tau^{<m}=\sum\left\{\tau^{n}\right.$ : $n<m\}$. Если $m=\aleph_{0}$, то положим $B(\tau)=B(\tau, m)$.

По построению:

- пространство $B(1)$ одноточечно;

- если $n \in \mathbb{N}$ и $n \geqslant 2$, то $B(n)$ есть канторово совершенное множество;

- пространство $B(\omega)=B\left(\aleph_{0}\right)$ гомеоморфно пространству иррациональных чисел $\mathbb{N}^{\mathbb{N}}$;

- $B(\tau)$ есть полное метризуемое пространство;

- $B(\tau, m)$ есть наследственно паракомпактное нульмерное пространство.

Обозначим через $K(\tau, m)$ совокупность всех кортежей длины меньше $m$. Тогда $|K(\tau, m)|=\tau^{<m}$.

Если $S \subseteq B(\tau, m)$, то для каждой $(\tau, m)$-таблицы множеств $\left\{E_{k}: k \in K(\tau, m)\right\}$ положим

$$
A_{S}\left\{E_{k}\right\}=\bigcup\left\{\bigcap\left\{E_{\xi \mid \beta}: \beta<m\right\}: \xi \in S\right\} .
$$

Операция $A_{S}$ является $\tau<m_{-} \delta s$-операцией типа $A$-операции.

Операция $(\tau, m)-A=A_{B(\tau, m)}$ назьвается $(\tau, m)$ - $A$-операцией. Операция $\tau-A=$ $\left(\tau, \aleph_{0}\right)$ - $A$ назьвается $\tau$ - $A$-операцией. Операция $A=\aleph_{0}-A$ назьвается $A$-операцией.

$A$-операция была введена П. С. Александровым [1], $\tau$ - $A$-операция - А. Стоуном [51] и $(\tau, \tau)$ - $A$-операция - Ю. С. Очаном [43].

3.1. ТеОремА. Пусть $S \subseteq B(\tau, m), \tau^{<m}=\tau$ и существует замкнутое подмножество $H \subseteq S$, которое непрерывно отображсается на $S^{m}$. Тогда операция $A_{S}$ нормальна.

Нормальность операции $(\tau, \tau)$ - $A$ была установлена Ю. С. Очаном, а нормальность операции $\tau$ - $A$ доказана А. Стоуном.

3.2. ТЕОРеМА. Пусть $X$ является нормальным счетнокомпактным пространством, $\left\{L_{n} \in A(X): n \in \mathbb{N}\right\} u \bigcap\left\{L_{n}: n \in \mathbb{N}\right\}=\varnothing$. Тогда справедливь следующие утвержсдения. 
1. Существует непрерывное отображение $g: X \rightarrow Y$ на метризуемый компакт $Y$ такое, что $\bigcap\left\{g\left(L_{m}\right): m \in \mathbb{N}\right\}=\varnothing$ и $g\left(L_{m}\right) \in A(Y)$ для всех $m \in \mathbb{N}$.

2. Существует последовательность бәровских подмножсеств $\left\{H_{n}: n \in \mathbb{N}\right\} \subseteq$ $B a(X)$ таких, что $\bigcap\left\{H_{n}: n \in \mathbb{N}\right\}=\varnothing u L_{m} \subseteq H_{m}$ для всех $m \in \mathbb{N}$.

ДоказАтельство. Существуют такие замкнутые в $X$ множества $\left\{E_{n_{1} \ldots n_{k}}^{m}: m, k\right.$, $\left.n_{1}, \ldots, n_{k} \in \mathbb{N}\right\}$, что $L_{m}=A\left\{E_{n_{1} \ldots n_{k}}^{m}\right\}$ для каждого $m \in \mathbb{N}$.

Рассмотрим минимальное семейство $\mathscr{L}$ замкнутых подмножеств пространства $X$, для которого:

- $E_{n_{1} \ldots n_{k}}^{m} \in \mathscr{L}$ для всех $m, k, n_{1}, \ldots, n_{k} \in \mathbb{N}$;

- если $L, H \in \mathscr{L}$, то $L \cap H \in \mathscr{L}$.

Можно считать, что $E_{n_{1} \ldots n_{k} n_{k+1}}^{m} \subseteq E_{n_{1} \ldots n_{k}}^{m}$ для всех $m, n_{1}, \ldots, n_{k+1} \in \mathbb{N}$.

Семейство $\mathscr{L}$ счетно. Пусть $\left\{\left(H_{n}, P_{n}\right): n \in \mathbb{N}\right\}$ состоит из всех пар множеств $(H, P)$, где $H, P \in \mathscr{L}$ и $H \cap P=\varnothing$. Для каждого $n \in \mathbb{N}$ сушествует непрерьвная функция $g_{n}: X \rightarrow[0,1]$ такая, что $H_{n} \subseteq g_{n}^{-1}(0)$ и $P_{n} \subseteq g_{n}^{-1}(1)$.

Пусть $g: X \rightarrow Y$ есть непрерьвное отображение такое, что $Y=g(X) \subseteq[0,1]^{\mathbb{N}}$ и $g(x)=\left(g_{n}(x): n \in \mathbb{N}\right)$ для каждой точки $x \in X$.

Множества $g\left(E_{n_{1} \ldots n_{k}}^{m}\right)$ замкнуты в $Y$ и $g\left(L_{m}\right)=A\left\{g\left(E_{n_{1} \ldots n_{k}}^{m}\right)\right\}$. Легко доказать, что $\bigcap\left\{g\left(L_{m}\right): m \in \mathbb{N}\right\}=\varnothing$. Утверждение 1 доказано. У тверждение 2 следует из утверждения 1 и известной теоремы П. С. Новикова [42] о кратной отделимости.

3.3. ТеОрема. Пусть $f: X \rightarrow Y$ есть непрерьвное отображение псевдокомпакта $X$ на несчетное метризуемое пространство $Y$. Тогда справедливы следующие утверждения.

1. $A(X) \backslash B a(X) \neq \varnothing$.

2. Cуществует $L \in A(X)$ такое, что $X \backslash L \notin A(X)$.

3. $\sigma_{\alpha}(Z(X)) \backslash \bigcup\left\{\sigma_{\beta}(Z(X)): \beta<\alpha\right\} \neq \varnothing$ дя каждого $\alpha>0$.

Теорема 3.3 установлена в [46], [53]. Для случая несчетного совершенно нормального компакта утверждение 1 было доказано А. В. Чернавским [8], а утверждение 3 В. И. Пономаревым [48].

3.4. ПроблемА (Р. Д. Маулдин, см. [49; проблема 12]). Пусть $X$ есть неразреженньй компакт. Верно ли, что $A(L(X)) \backslash B o(X) \neq \varnothing$ и $B o(X) \backslash B_{\alpha}(X) \neq \varnothing$ для всех $\alpha<\omega_{1}$ ?

\section{4. Общая характеристика $A$-множеств}

Пространство $X$ назьвается $P^{\tau}$-пространством, если пересечение любого семейства из не более $\tau$ открытых множеств является открытьм множеством. Пространство называется $P^{<\tau}$-пространством, если оно является $P^{m}$-пространством для каждого $m<\tau$. Если $\tau=\aleph_{0}$, то любое пространство является $P^{<\tau}$-пространством.

4.1. ТЕОРЕМА. Пусть $\tau$ и ми, $\tau^{<m}=\tau$ и $S \subseteq B(\tau, m)$. Тогда для любого $P^{<\tau}$-пространства $X$ и его подмножества $E \subseteq X$ справедливы следующие утверждения.

1. $E \in A_{S}(X)$ тогда и только тогда, когда существует такое замкнутое в $X \times S \subseteq X \times B(\tau, m)$ множество $H$, что $p_{X}(H)=E$, где $p_{X}(x, s)=x$ для всех $(x, s) \in X \times S$. 
2. $E \in A_{S}(\mathscr{U}(X))$ тогда и только тогда, когда существует такое $G_{\tau}$-множество $M$ пространства $X \times S$, что $p_{X}(M)=E$.

3. $E \in A_{S}(L(X))$ тогда и только тогда, когда существуют замкнутое в $X \times S$ множество $H$ и $G_{\tau}$-множество $M$ в $X \times S$, для которых $E=p_{X}(H \cap M)$.

ДокАЗАтельство. Пусть заданы замкнутые в $X$ множества $\left\{F_{k}: k \in K(\tau, m)\right\}$ и открытые в $X$ множества $\left\{V_{k}: k \in K(\tau, m)\right\}$, для которых $E=A_{S}\left\{F_{k} \cap V_{k}\right\}=$ $\bigcup\left\{\cap\left\{F_{\xi \mid \beta} \cap V_{\xi \mid \beta}: \beta<m\right\}: \xi \in S\right\}$. Положим $H_{\beta}=\bigcup\left\{F_{\xi \mid \beta} \times \delta(\xi \mid \beta): \xi \in B(\tau, m)\right\}$, $M_{\beta}=\bigcup\left\{V_{\xi \mid \beta} \times \delta(\xi \mid \beta): \xi \in B(\tau, m)\right\}, H=\bigcap\left\{(X \times S) \cap H_{\beta}: \beta<m\right\}$ и $M=$ $\bigcap\left\{(X \times S) \cap M_{\beta}: \beta<m\right\}$. Тогда $E=p_{X}(H \cap M)$. Этим мы представили подмножество $E \in A_{S}(L(X))$ как проекцию пересечения замкнутого множества и $G_{\tau}$-множества из $X \times S$. При $V_{k}=X$ мы получаем представление множества $E \in A_{S}(\mathscr{F}(X))$ в виде проекции замкнутого в $X \times S$ множества $H$. При $F_{k}=X$ мы представляем $E \in A_{S}(\mathscr{U}(X))$ в виде проекции $G_{\tau}$-множества $M$.

В одном направлении утверждения теоремы доказаны. Эти импликации верны для любого пространства $X$, т.е. требование быть $P^{<\tau}$-пространством не существенно.

Пусть множество $H$ замкнуто, а множества $\left\{W_{\alpha}: \alpha \in J_{m}\right\}$ открыты в $X \times S$. Предположим, что $W=\bigcap\left\{W_{\alpha}: \alpha \in J_{m}\right\}, p_{X}(H \cap W)=E$ и $W_{\alpha} \subseteq W_{\beta}$ при $\beta<\alpha$.

По индукции строятся отображение $h: K(\tau, m) \rightarrow K(\tau, m)$, открытые в $X$ множества $\left\{V_{k}: k \in K(\tau, m)\right\}$ и открытые в $B(\tau, m)$ множества $\left\{H_{k}: k \in K(\tau, m)\right\}$, для которых:

1) $H_{k}=\delta_{h(k)} \cap S$ для любого $k \in K(\tau, m)$;

$2)$ если $\xi=\left(\xi_{\alpha}: \alpha<m\right)$ и $1 \leqslant \beta<\mu<m$, то сушествуют $\eta=\left(\eta_{\alpha}: \alpha<m\right)$ и $1 \leqslant \beta^{\prime}<\mu^{\prime}<m$, для которых $h(\xi \mid \beta)=\eta \mid \beta^{\prime}$ и $h(\xi \mid \mu)=\eta \mid \mu^{\prime} ;$

3) $W_{1}=\bigcup\left\{V_{\alpha} \times H_{\alpha}: \alpha \in J_{\tau}\right\}$;

4) если $k=\left(k_{\alpha}: \alpha<\beta\right)$, то $W_{\beta} \cap\left(V_{k} \times H_{k}\right)=\bigcup\left\{V_{\left(k_{1} \ldots k_{\alpha} \ldots \mu\right)} \times H_{\left(k_{1} \ldots k_{\alpha} \ldots \mu\right)}\right.$ : $\left.\mu \in J_{\tau}\right\}$.

Пусть $F_{k}=\operatorname{cl}_{X} p_{X}\left(H \cap\left(X \times H_{k}\right)\right)$ и $E_{k}=F_{k} \cap V_{k}$. Тогда $E=A_{S}\left\{E_{k}\right\}$. Доказательство завершено.

При $\tau=\aleph_{0}$ и $S=B(\tau)$ утверждения 1 и 2 доказаны в [11].

При $m=\tau=\aleph_{0}$ и $S=B(\omega)$ утверждение 1 доказано Н. Н. Лузиным (см. [23], [28]), а утверждение 3 доказано в [21].

Этим же способом устанавливается приводимая ниже теорема.

4.2. Теорема. Пусть $X$ есть топологическое пространство и $E \subseteq X$. Тогда справедливы следующие утверждения.

1. Если $E \in B o(X)$, то существуют замкнутые в $X \times B(\omega)$ множество $H$ и $G_{\delta}$-множество $M \subseteq X \times B(\omega)$, для которых $E=p_{X}(H \cap M)$ и множество $(\{x\} \times B(\omega)) \cap H \cap M$ одноточечно для каждой точки $x \in E$.

2. Если пространство $X$ совершенно нормально и $E \in B o(X)$, то существуют замкнутое в $X \times B(\omega)$ множество $H$ и $G_{\delta}$-множество $M \subseteq X \times B(\omega)$, для которых $E=p_{X}(M)=p_{X}(H)$ и множества $(\{x\} \times B(\omega)) \cap H u(\{x\} \times B(\omega)) \cap M$ одноточечны для каждой точки $x \in E$.

4.3. ЗАмЕчАнИЕ. Если $M \subseteq X \times B(\tau)$ и множество $(\{x\} \times B(\tau)) \cap M$ одноточечно для всех $x \in p_{X}(M)$, то говорят, что множество $M$ униформно. 


\section{5. Абсолютные $\Psi$-множества}

Пусть задано бесконечное кардинальное число $\tau$. Рассматриваем только $\tau$-st-операции.

5.1. ОПРЕДЕЛЕНИЕ. Пусть $\Psi$ является $\tau$-st-операцией. Пространство $X$ назьвается:

- абсолютным $\Psi$-множеством, если существует компактное пространство $Y$ для каждого $X \in \Psi(\mathscr{F}(Y))$;

- слабо абсолютным $\Psi$-множеством, если $X \in \Psi(\mathscr{F}(Y))$ для некоторого полного в смысле Чеха пространства $Y$;

- $\mathscr{U}$-абсолютным $\Psi$-множеством, если $X \in \Psi(\mathscr{U}(Y))$ для некоторого компакта;

- слабо $\mathscr{U}$-абсолютным $\Psi$-множеством, если $X \in \Psi(\mathscr{U}(Y))$ для некоторого полного в смысле Чеха пространства $Y$;

- $L$-абсолютным $\Psi$-множеством, если $X \in \Psi(L(Y))$ для некоторого компакта;

- слабо $L$-абсолютным $\Psi$-множеством, если $X \in \Psi(L(Y))$ для некоторого полного в смысле Чеха пространства $Y$.

Аналогичным образом определяются абсолютные, слабо абсолютные, $\mathscr{U}$-абсолютные и $L$-абсолютные $\Psi$-множества заданного класса $\alpha<\tau^{+}$.

5.2. ТеоремА. Для пространства $X$ следующие утверждения равносильньь.

1. $X$ является абсолютным $\Psi$-множеством для некоторой $\tau$-st-операичи $\Psi$.

2. $X$ является слабо абсолютным $\Psi$-множеством для некоторой $\tau$-st-операиии $\Psi$.

3. $X$ является $\mathscr{U}$-абсолютным $\Psi$-множеством для некоторой $\tau$-st-операичи $\Psi$.

4. $X$ является слабо $\mathscr{U}$-абсолютным $\Psi$-множеством для некоторой $\tau$-stоперации $\Psi$.

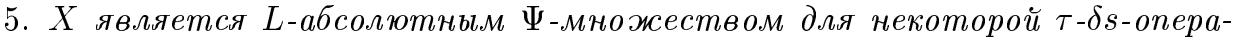
иич $\Psi$.

6. $X$ является слабо L-абсолютным $\Psi$-множеством для некоторой $\tau$-stоперачии $\Psi$.

ДокАЗАТЕЛЬСТВо. Импликации $1 \Rightarrow 2 \Rightarrow 6,3 \Rightarrow 4 \Rightarrow 6$ и $5 \Rightarrow 6$ очевидны.

Рассмотрим $\tau$-базу $S$. Предположим, что $X$ является подпространством пространства $Y, \Psi=\Psi_{S}, E_{\alpha} \subseteq Y$ для всех $\alpha \in J_{\tau}$ и $X=\Psi\left\{E_{\alpha}\right\}$.

Если $\xi \in J_{\tau}$, то положим $\bar{\xi}=J_{\tau} \backslash \xi$. Пусть $\bar{S}=\{\bar{\xi}: \xi \in S\}$ и $H_{\alpha}=Y \backslash E_{\alpha}$. Тогда $\Psi_{\bar{S}}\left\{H_{\alpha}\right\}=\Psi_{S}\left\{E_{\alpha}\right\}=X$. Этим доказаны импликации $1 \Rightarrow 3 \Rightarrow 1$ и $2 \Rightarrow 4 \Rightarrow 2$.

Предположим, что $Y$ является полным по Чеху пространством, $Z=\beta Y$ и $Y=$ $\bigcap\left\{W_{n}: n \in \mathbb{N}\right\}$, где множества $W_{n}$ открыты в $Z$. Пусть $E_{\alpha} \in \mathscr{F}(Y), V_{\alpha} \in \mathscr{U}(Y)$ и $X=\Psi\left\{E_{\alpha} \cap V_{\alpha}\right\}$, где $\Psi=\Psi_{S}$. Разбиваем $J_{\tau}$ на три попарно дизъюнктных подмножества $M, P$ и $Q$, для которых:

1) множество $M$ счетно и $M=\left\{\mu_{n}: n \in \mathbb{N}\right\}$;

2) сушествуют взаимно однозначные отображения $p: J_{\tau} \rightarrow P$ и $d: J_{\tau} \rightarrow Q$.

Если $\mu \in M$ и $\mu=\mu_{n}$, то $F_{\mu}=Z \backslash W_{n}$. Если $\mu \in P$, то $F_{\mu}=\operatorname{cl}_{Z} E_{p^{-1}(\mu)}$. Если $\mu \in Q$, то $F_{\mu}=\operatorname{cl}_{Z}\left(Y \backslash V_{q^{-1}(\mu)}\right)$. Если $\xi \subseteq J_{\tau}$, то $S(\xi)=\left\{\eta \subseteq J_{\tau}: \eta \subseteq P \cup Q\right.$, 
$\left.p(\xi) \subseteq \eta \subseteq J_{\tau} \backslash q(\xi)\right\}$. Если $S_{1}=\bigcup\{S(\xi): \xi \in S\}$, то $X=\Psi_{S_{1}}\left\{F_{\mu}\right\}$. Импликация $6 \Rightarrow 1$ и утверждения теоремы доказаны.

Таким образом, понятия слабого абсолютного $\Psi$-множества, $\mathcal{U}$-абсолютного и $L$-абсолютного $\Psi$-множества имеют смысл для $\tau$ - $\delta s$-операций.

Легко доказываются следующие утверждения.

5.3. ПРЕДЛОЖЕНИЕ. Пространство $X$ является абсолютным $\Psi$-множеством для некоторой $\tau$-st-операции $\Psi$ тогда и только тогда, когда существует семейство замкнутых в $\beta X$ множеств $\left\{F_{\alpha}: \alpha \in J_{\tau}\right\}$ maких, что $\left(\bigcap\left\{F_{\alpha}: x \in\right.\right.$ $\left.\left.F_{\alpha}\right\}\right) \cap\left(\bigcap\left\{\beta X \backslash F_{\beta}: x \notin F_{\beta}\right\}\right) \subseteq X$ для каждой точки $x \in X$.

5.4. ПРЕДЛОЖЕНИЕ. Пространство $X$ является абсолютныл $\Psi$-множеством для некоторой $\tau$ - $\delta$-операции тогда и только тогда, когда существует семейство замкнутых в $\beta X$ множсеств $\left\{F_{\alpha}: \alpha \in J_{\tau}\right\}$ таких, что $\left\{\left\{F_{\alpha}: x \in\right.\right.$ $\left.F_{\alpha}\right\} \subseteq X$ для каждой точки $x \in X$.

5.5. ПРЕДЛОЖЕНИЕ. Пусть $X$ является абсолютным $\Psi$-множеством для некоторой $\tau-\delta$-операции $\Psi$. Тогда любое открытое покрытие пространства $X$ содержит подпокрытие мощности $\leqslant \tau$.

5.6. ПРЕДЛОЖЕНИЕ. Пусть $f: X \rightarrow Y$ есть совершенное отображсение пространства $X$ на пространство $Y$. Пространство $X$ является абсолютным $\Psi$-множеством для некоторой $\tau$ - $\delta$-операции $\Psi$ тогда и только тогда, когда $Y$ является $\Phi$-множеством для некоторой $\tau-\delta s$-операции $\Phi$.

Из теоремы 4.1 вытекает следующее утверждение.

5.7. СлЕДСТВИЕ. Пространство $X$ является L-абсолютным $\tau$-A-множеством тогда и только тогда, когда существует такое полное в смисле Чеха подпространство $H \subseteq X \times B(\tau)$, что $X=p_{X}(H)$.

\section{6. Абсолютные $A_{S}$-множества}

Фиксируем бесконечные кардинальные числа $\tau, m$ и непустое подмножество $S \subseteq$ $B(\tau, m)$.

6.1. ТЕОРЕМА. Пусть $\Psi=A_{S}$ и $X$ является подпространством пространства $Y$.

1. Если $X$ является абсолютным $\Psi$-множеством, то $X \in \Psi(\mathscr{F}(Y))$.

2. Если $X$ является слабо абсолютным $\Psi$-множеством, то существуют $G_{\delta}$-множсество $Z$ пространства $Y$ и абсолютное $\Psi$-множсество $P \in \Psi(\mathscr{F}(\beta Y))$, для которых $X \in \Psi(\mathscr{F}(Z)) u X=Z \cap P$.

ДоКАЗАТЕльСТво. Можно предположить, что $X$ всюду плотно в $Y$. Пусть $X$ является всюду плотным подпространством пространства $X_{1}$ и $X \in \Psi\left(\mathscr{F}\left(X_{1}\right)\right)$. Тогда $X=\Psi\left\{F_{k}\right\}$, где $\left\{F_{k}: k \in K(\tau, m)\right\}$ есть семейство замкнутых в $X_{1}$ множеств. Можем считать, что $F_{\xi \mid \alpha} \subseteq F_{\xi \mid \beta}$ при $\beta \leqslant \alpha<m$.

Сушествует непрерьвное отображение $f: \beta X \rightarrow \beta X_{1}$ такое, что $f(x)=x$ для всех $x \in X$. Положим $X_{2}=f^{-1}\left(X_{1}\right)$ и $E_{k}=f^{-1}\left(F_{k}\right)$. Тогда $X=\Psi\left\{E_{k}\right\}$ и $X \in \Psi\left(\mathscr{F}\left(X_{2}\right)\right)$. Сушествует непрерьвное отображение $g: \beta X \rightarrow \beta Y$ такое, что $g(x)=x$ для всех $x \in X$. 
Если пространство $X_{1}$ компактно, то $X_{2}=\beta X$. В этом случае положим $\Phi_{k}=$ $Y \cap g\left(E_{k}\right)$. Тогда $X=\Psi\left\{\Phi_{k}\right\}$ и утверждение 1 доказано.

Пусть $X_{1}$ является полным по Чеху пространством. Тогда сушествует такое $G_{\delta^{-}}$ множество $Z$ пространства $\beta Y$, что $X \subseteq Z \subseteq Y$ и $g^{-1}(Z) \subseteq X_{2}$. Положим $\Phi_{k}=$ $\operatorname{cl}_{\beta Y} g\left(E_{k}\right)$ и $H_{k}=Z \cap g\left(E_{k}\right)=Z \cap \Phi_{k}$. Тогда $X=\Psi\left\{H_{k}\right\}$. Если $P=\Psi\left\{\Phi_{k}\right\}$, то $X=Z \cap P$. Утверждение 2 и теорема доказаны.

6.2. СлЕДСТВИЕ. Если $X$ является слабо абсолютным $\tau$-А-множеством $и$ $Z$ является компактификачией пространства $X$, то $X$ является пересечением $\tau$-A-множества и $G_{\delta}$-множества пространства $Z$.

Следствие 6.2 решает проблему 39 А. Стоуна из [49].

\section{7. Ядра отображений}

Скажем, что пространство $Z$ является ядром отображения $f: X \rightarrow Y$, если существует вложение $\phi: X \rightarrow Y \times Z$ такое, что $f(x)=p_{Y}(\phi(x))$ для всех $x \in X$, где $p_{Y}(y, z)=y$ для $(y, z) \in Y \times Z$.

Ясно, что ядра существуют только для непрерывных отображений.

Отображение $f: X \rightarrow Y$ назьвается

- отображением с $\tau$-ядром, если для $f$ сушествует метризуемое ядро веса $\tau$;

- отображением с сепарабельным ядром, если для $f$ сушествует метризуемое сепарабельное ядро;

- $s(\tau)$-отображением, если каждый прообраз $f^{-1}(y)$ является подпространством веса $\leqslant \tau$;

- s-отображением, если каждый прообраз $f^{-1}(y)$ является сепарабельньм метризуемьг подпространством.

Каждое отображение с $\tau$-ядром является $s(\tau)$-отображением. Обратное утверждение неверно. Взаимно однозначное отображение $f: X \rightarrow Y$ дискретного пространства $X$ на несчетньй метризуемый компакт $Y$ является $s$-отображением и не имеет сепарабельного ядра.

7.1. ПРЕДЛОЖЕНИЕ. Пусть $f: X \rightarrow Y$ является открытым непрерывным отображсением пространства $X$ на пространство $Y, \tau$ является бесконечным кардиналом и равномерные веса пространств $X$ и $Y$ не превосходят $\tau$.

Тогда следующие утверждения равносильнь.

1. $f$ является отображсением $с$ ядром веса $\leqslant \tau$.

2. На Х существуют открытые дискретные семейства $\left\{\gamma_{\alpha}=\left\{U_{\alpha \beta}: \beta \in B_{\alpha}\right\}\right.$ : $\alpha \in A\}$ такие, что $|A| \leqslant \tau$, семейство $\left\{U_{\alpha \beta}: \beta \in B_{\alpha}, \alpha \in A\right\}$ образует базу пространства $X$ и для каждого $\alpha \in A$ семейство $f\left(\gamma_{\alpha}\right)=\left\{f\left(U_{\alpha \beta}\right)\right.$ : $\left.\beta \in B_{\alpha}\right\}$ дискретно в $Y$.

3. Все прообразы $f^{-1}(y), y \in Y$, являются подпространствами веса $\leqslant \tau$.

ДокАЗАТЕльство. Пространство $Z$ имеет равномерный вес не больше $\tau$ тогда и только тогда, когда вьполняются условия:

1) в $Z$ сушествует $\tau$-дискретная база, т.е. база, которая распадается в объединение $\tau$ дискретных систем;

2 ) для каждого открытого в $Z$ множества $V$ существует семейство непрерьвных функций $\left\{f_{\alpha}: Z \rightarrow[0,1]: \alpha \in J_{\tau}\right\}$ таких, что $V=\bigcup\left\{f_{\alpha}^{-1}(1): \alpha \in J_{\tau}\right\}=$ $\bigcup\left\{f^{-1}(0,2): \alpha \in J_{\tau}\right\}$. 
Импликация $1 \Rightarrow 2$ очевидна. Если $\left\{V_{\mu}: \mu \in M\right\}$ есть $\tau$-дискретная база пространства $Y$ и $\left\{W_{\xi}: \xi \in J_{\tau}\right\}$ есть база пространства $Z$, то $\left\{V_{\mu} \times W_{\alpha}: \mu \in M, \alpha \in J_{\tau}\right\}$ есть $\tau$-дискретная база пространства $Y \times Z$.

Импликация $2 \Rightarrow 3$ также очевидна.

Если $y \in Y$, то множество $B_{\alpha}(y)=\left\{\beta \in B_{\alpha}: U_{\alpha \beta} \cap f^{-1}(y) \neq \varnothing\right\}$ не более чем одноточечно и $\left\{U_{\alpha \beta} \cap f^{-1}(y): \beta \in B_{\alpha}(y), \alpha \in A\right\}$ образует базу пространства $f^{-1}(y)$.

Пусть веса прообразов $f^{-1}(y)$ не превосходят $\tau$. На $X$ фиксируем дискретные семейства $\left\{\gamma_{\alpha}=\left\{W_{\mu}: \mu \in M_{\alpha}\right\}: \alpha \in J_{\tau}\right\}$, для которых $\left\{W_{\mu}: \mu \in M_{\alpha}, \alpha \in J_{\tau}\right\}$ является базой. На $Y$ фиксируем семейства дискретных семейств $\left\{\omega_{\alpha}=\left\{V_{\eta}: \eta \in P_{\alpha}\right\}\right.$ : $\left.\alpha \in J_{\tau}\right\}$, для которых $\left\{V_{\eta}: \eta \in P_{\alpha}, \alpha \in J_{\tau}\right\}$ является базой. Для любых $\alpha, \beta \in J_{\tau}$ и $\eta \in P$ множество $M(\alpha, \beta, \eta)=\left\{\mu \in M_{\alpha}: V_{\eta} \subseteq f\left(W_{\mu}\right)\right\}$ имеет мошность $\leqslant \tau$. Если $M(\alpha, \beta, \eta)=\varnothing$, то $H_{\alpha \beta \mu \eta}=\varnothing$ для всех $\mu \in J_{\tau}$. Если $M(\alpha, \beta, \eta) \neq \varnothing$, то фиксируем отображение $h$ множества $J_{\tau}$ на $M(\alpha, \beta, \eta)$ и положим $H_{\alpha \beta \mu \eta}=W_{f(\mu)} \cap f^{-1}\left(V_{\eta}\right)$. Пусть $\xi_{\alpha \beta \mu}=\left\{H_{\alpha \beta \mu \eta}: \eta \in P_{\beta}\right\}$. Тогда семейства $\xi_{\alpha \beta \mu}$ и $f\left(\xi_{\alpha \beta \mu}\right)$ дискретны и $\left\{H_{\alpha \beta \mu \eta}: \eta \in P_{\beta}, \alpha, \beta, \mu \in J_{\tau}\right\}$ является базой пространства $X$. Импликация $3 \Rightarrow 2$ доказана.

Рассмотрим на $X$ открытые дискретные семейства $\left\{\gamma_{\alpha}=\left\{U_{\alpha \beta}: \beta \in B_{\alpha}\right\}: \alpha \in A\right\}$ такие, что $|A|=\tau$, семейство $\left\{U_{\alpha \beta}\right\}$ образует базу и семейство $f\left(\gamma_{\alpha}\right)$ дискретно в $Y$ для каждого $\alpha \in A$. Для каждого $\alpha \in A$ положим $U_{\alpha}=\bigcup\left\{U_{\alpha \beta}: \beta \in B_{\alpha}\right\}$. Сушествует семейство непрерьвных функций $\left\{f_{\alpha \beta}: X \rightarrow I_{\alpha \beta}=[0,1]: \beta \in A\right\}$, для которых $U_{\alpha}=\bigcup\left\{f_{\alpha \beta}^{-1}(0,2): \beta \in A\right\}=\bigcup\left\{f_{\alpha \beta}^{-1}(1): \beta \in A\right\}$. Пусть $Z=\prod\left\{I_{\alpha \beta}: \alpha, \beta \in A\right\}$. Рассмотрим топологическое вложение $g: X \rightarrow Y \times Z$, где $g(x)=\left(f(x), f_{\alpha \beta}(x)\right.$ : $\alpha, \beta \in A)$. Итак, $Z$ является ядром веса $\tau$ отображения $f$. Импликация $2 \Rightarrow 1$ и предложение доказаны.

7.2. СледСтвиЕ (А. Окуяма, М. Чобан, см. [5]). Пусть $f: X \rightarrow Y$ является открытымм непрерывным s-отображением метризуемого пространства $X$ на $Y$, $g: Y \rightarrow Z$ является открытыцм непрерывным отображением на метризуемое пространство $Z$ и все прообразы $g^{-1}(z)$ сепарабельны. Тогда пространство $Y$ метризуемо.

Аналогичным образом устанавливается справедливость следующего утверждения.

7.3. ПРЕДЛОЖЕНИЕ. Пусть $f: X \rightarrow Y$ является открытылм непрерывныцм s-отображением метризуемого пространства $X$ на $Y$.

Следующие утверждения равносильны.

1. Отображение $f$ имеет сепарабельное ядро.

2. Пространство $Y$ имеет б-дизбюнктную базу.

7.4. СлЕдСтвиЕ. Если $f: X \rightarrow Y$ является открытым отображением с сепарабельныц ядром метризуемого пространства $X$ на совершенно нормальное пространство $Y$, то пространство $Y$ метризуемо.

\section{8. Проблема Хаусдорфа об открытых отображениях. Общий случай}

Ф. Хаусдорф в 1934 г. (см. [20]) доказал, что открытый непрерьвньй образ сепарабельного полного метрического пространства является полным метризуемым пространством. 
Полные метризуемые пространства являются абсолютными $G_{\delta}$-пространствами, т.е. абсолютно борелевскими множествами класса 1 . В этом контексте $\Phi$. Хаусдорф сформулировал следуюшую проблему.

8.1. ПроБлЕма ХАУсдОРфА. Пусть $f: X \rightarrow Y$ является открытым непрерьвньм отображением метризуемого сепарабельного пространства $X$ на $Y$. Пусть $X$ является абсолютным борелевским множеством класса $\alpha \geqslant 2$. Будет ли $Y$ абсолютно борелевским множеством класса $\leqslant \alpha$ ?

Ослабленным вариантом проблемы Хаусдорфа является следующая проблема.

8.2. ПроБлемА. Пусть $f: X \rightarrow Y$ является открытым $s$-отображением метризуемого слабо абсолютно борелевского множества $X$ на метризуемое пространство $Y$. Будет ли $Y$ слабо абсолютно борелевским множеством?

8.3. ТеоремА. Пусть Y есть метризуемое пространство. Следуюшие утверждения равносильны.

1. $Y$ есть слабо абсолютное А-мнохсество.

2. $Y$ есть открытый непрерывныц $s$-образ некоторого метризуемого слабо абсолютного А-множества.

3. $Y$ есть открытый непрерывный s-образ некоторого метризуемого слабо

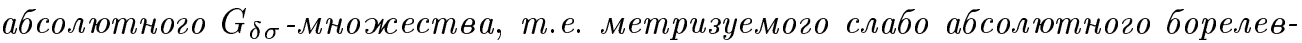
ского множества второго класса.

ДоКАЗАТЕЛЬСТВО. Импликации $3 \Rightarrow 2$ и $1 \Rightarrow 2$ очевидны.

Пусть $f: X \rightarrow Y$ является непрерьвньм открытым $s$-отображением метризуемого слабо абсолютного $A$-множества $X$ на $Y$. В силу предложения 7.1 можно считать, что существуют полное метризуемое сепарабельное пространство $Z$ и полное метризуемое пространство $Y_{1}$ такие, что $Y$ является подпространством пространства $Y_{1}, X$ является подпространством пространства $Y \times Z$ и $f(y, z)=y$ для всех $x=(y, z) \in X$. Ясно, что $X$ является $A$-множеством пространства $Y \times Z$. Поскольку пространство $B(\omega)$ допускает открытое непрерьвное отображение на $Z$, то существует $A$-множество $X_{1} \subseteq Y_{1} \times B(\omega)$, которое проектируется на $Y$. В силу теоремы 3.4 $Y$ является $A$-множеством пространства $Y_{1}$. Импликашия $2 \Rightarrow 1$ доказана.

Пусть $Y$ является $A$-множеством полного метрического пространства $Y_{1}$. В силу теоремы 3.4 сушествует замкнутое в $Y_{1} \times B(\omega)$ множество $\Phi$, которое проектируется на $Y$. Пусть $\left\{U_{n}: n \in \mathbb{N}\right\}$ есть база пространства $B(\omega)$ из открыто-замкнутых подмножеств. Для каждого $n \in \mathbb{N}$ в $Y_{1} \times U_{n}$ сушествует замкнутое подмножество $X_{n}$, которое проектируется на $Y$. Положим $X=\bigcup\left\{X_{n}: n \in \mathbb{N}\right\}$ и $f(y, z)=y$ для всех $x=(y, z) \in X$. Тогда $X$ является $G_{\delta \sigma}$-множеством пространства $Y_{1} \times B(\omega)$, а $f$ является открытым $s$-отображением пространства $X$ на $Y$. Импликашия $1 \Rightarrow 3$ и теорема доказаны.

При помощи теоремы 3.4 аналогичным образом доказывается следующая теорема.

8.4. ТЕОремА. Для каждого метризуемого слабо абсолютного борелевского множсества $Y$ существуют метризуемое слабо абсолютное $G_{\delta \sigma}$-мнохсество $X$ u непрерывное открытое счетнократное отображсение $f: X \rightarrow Y$ на $Y$.

Еше Н. Н. Лузиным [36], [37] было установлено, что непрерьвньй образ абсолютного $A$-множества является абсолютным $A$-множеством. Поэтому весьма неожиданньм оказалось следуюшее утверждение. 
8.5. Теорема (Л.В. Келдьш [33]). Каждое метризуемое абсолютное А-множество является открытым непрерывным образом метризуемого абсолютного $G_{\delta \sigma}$-множества.

Теорема 8.5 является следствием теоремы 8.3. Этим установлено, что в общем случае проблемы Хаусдорфа 8.1 и 8.2 имеют отрищательное решение. Поэтому представляет особый интерес следуюшая проблема.

8.6. ПроБлЕмА. При каких дополнительных условиях ответына вопросы из проблем Хаусдорфа положительны?

\section{9. Проблема Хаусдорфа. Случай равномерно полных отображений}

Отображение $f: X \rightarrow Y$ пространства $X$ на $Y$ равномерно полно, если сушествует такое полное в смысле Чеха пространство $X^{\prime}$, что $X$ является подпространством пространства $X^{\prime}$ и каждый прообраз $f^{-1}(y), y \in Y$, замкнут в $X^{\prime}$. Всегда можем считать, что $X \subseteq X^{\prime} \subseteq \beta X$.

Если пространство $X$ метризуемо, то отображение $f: X \rightarrow Y$ равномерно полно тогда и только тогда, когда пространство $X$ метризуемо такой метрикой $\rho$, для которой все прообразы $f^{-1}(y), y \in Y$, полны в метрике $\rho$.

Различные понятия полноты отображения были рассмотрены в работах [46], [14], [39], [40], [56].

Пространство $Z$ является паракомпактньм $p$-пространством, если сушествует совершенное отображение пространства $Z$ на некоторое метризуемое пространство (А.В. Архангельский [4]).

9.1. ПреДлОЖЕНИЕ. Пусть $f: Z \rightarrow Y$ является непрерывным отображсением, $X_{1}$ является $G_{\delta}$-подмножеством пространства $\beta Z, X$ является паракомпактным р-подпространством пространства $Z, X \subseteq X_{1}, f(X)=Y$, $g=f \mid X: X \rightarrow Y$ является открытым отображением и для каждого у $\in Y$ множество $g^{-1}(y)$ замкнуто в $X_{1}$. Тогда $X$ является $G_{\delta}$-подмножеством пространства $Z$.

ДокАЗАТЕЛЬСТво. Можем считать, что пространство $X$ всюду плотно в $Z$. Тогда $X$ всюду плотно и в $X_{1}$.

Пусть $X_{1}=\bigcap\left\{V_{n}: n \in \mathbb{N}\right\}$, где множество $V_{n}$ открыто в $\beta X_{1}$ и $V_{n+1} \subseteq V_{n}$ для каждого $n \in \mathbb{N}$. Тогда сушествуют последовательность $\left\{\gamma_{n}=\left\{U_{\alpha}: \alpha \in A_{n}\right\}\right.$ : $n \in \mathbb{N}\}$ семейств из открытых в $\beta Z$ подмножеств и последовательность отображений $\left\{\pi_{n}: A_{n+1} \rightarrow A_{n}: n \in \mathbb{N}\right\}$, для которых:

1) если $U_{n}=\bigcup\left\{U_{\alpha}: \alpha \in A_{n}\right\}$, то $X \subseteq U_{n} \subseteq V_{n}$ для всякого $n \in \mathbb{N}$;

2) если $n \in \mathbb{N}$ и $\alpha \in A_{n}$, то $U_{\alpha} \cap X=\bigcup\left\{U_{\beta} \cap X: \beta \in \pi_{n}^{-1}(\alpha)\right\} \subseteq \bigcup\left\{\operatorname{cl}_{\beta Z} U_{\beta}\right.$ : $\left.\beta \in \pi_{n}^{-1}(\alpha)\right\} \subseteq U_{\alpha} \subseteq \operatorname{cl}_{\beta Z} U_{\alpha} \subseteq V_{n}$ и $U_{\alpha} \cap X$ является $F_{\sigma}$-подмножеством пространства $X$;

3) для любых $n \in \mathbb{N}$ и $x \in U_{n}$ существует открытое в $\beta Z$ множество $V$ такое, что множество $\left\{\alpha \in A_{n}: V \cap U_{\alpha} \neq \varnothing\right\}$ конечно и $x \in V$;

4) если $x \in X$ и $\gamma_{n}(x)=\bigcup\left\{U \in \gamma_{n}: x \in U\right\}$, то $\left\{\left\{\operatorname{cl}_{\beta Z} \gamma_{n}(x): n \in \mathbb{N}\right\} \subseteq X\right.$;

5) если $n \in \mathbb{N}, \alpha \in A_{n}$ и $\beta \in \pi_{n}^{-1}(\alpha)$, то $\operatorname{cl}_{Y}\left(f\left(U_{\beta} \cap X\right)\right) \subseteq f\left(U_{\alpha} \cap X\right)$.

Мы утверждаем, что $X=\bigcap\left\{U_{n} \cap Z: n \in \mathbb{N}\right\}$.

Предположим, что $x \in \bigcap\left\{U_{n} \cap Z: n \in \mathbb{N}\right\}$. 
Для каждого $n \in \mathbb{N}$ в силу условия 3 множество $A_{n}(x)=\left\{\alpha \in A_{n}: x \in U_{\alpha}\right\}$ конечно и $\pi_{n}\left(A_{n+1}(x)\right) \subseteq A_{n}(x)$. Рассматривая $A_{n}(x)$ как дискретные конечные пространства, получаем, что обратный предел $A=\lim \left\{A_{n}(x): \pi_{n} \mid A_{n+1}(x)\right\}$ является непустым компактом.

Фиксируем точку $\alpha=\left(\alpha_{n} \in A_{n}(x): n \in \mathbb{N}\right) \in A$. По построению $x \in U_{\alpha_{n+1}} \subseteq$ $\operatorname{cl}_{\beta Z} U_{\alpha_{n+1}} \subseteq U_{\alpha_{n}} \subseteq U_{n} \subseteq V_{n}$ и $\alpha_{n}=\pi_{n}\left(\alpha_{n+1}\right)$ для всех $n \in \mathbb{N}$. Пусть $y=f(x)$. Множество $F=\bigcap\left\{U_{\alpha_{n}}: n \in \mathbb{N}\right\}$ является непустым компактным подмножеством пространства $X_{1}$, и для любого открытого в $\beta Z$ множества $V$, содержащего $F$, сушествует $n \in \mathbb{N}$ такое, что $U_{\alpha_{n}} \subseteq V$. Предположим, что $F \cap g^{-1}(y)=\varnothing$. Тогда сушествует $m \in \mathbb{N}$, для которого $U_{\alpha_{m}} \cap g^{-1}(y)=\varnothing$. В этом случае $y \notin f\left(U_{\alpha_{m}} \cap X\right)$ и $y \notin \operatorname{cl}_{Y} f\left(U_{\alpha_{m+1}} \cap X\right)$. Тогда $U_{\alpha_{m+1}} \cap Z \subseteq f^{-1}\left(f\left(U_{\alpha_{m+1}} \cap X\right)\right)$ и $U_{\alpha_{m+1}} \cap f^{-1}(y)=\varnothing$, противоречие с условием $x \in U_{\alpha_{m}} \cap f^{-1}(y)$. Итак, $F \cap g^{-1}(y) \neq \varnothing$. Фиксируем $x \in F \cap g^{-1}(y)$. Тогда $x \in U_{\alpha_{m}} \subseteq \gamma_{n}\left(x_{1}\right)$ для всех $n \in \mathbb{N}_{\text {и }} x \in \bigcap\left\{\gamma_{k}\left(x_{1}\right): k \in \mathbb{N}\right\} \subseteq X$. Доказательство завершено.

9.2. ТеОРемА. Пусть $g: X \rightarrow Y$ является открытым непрерывнылм равномерно полным отображсением метризуемого пространства $X$ на паракомпакт $Y$ и $\alpha \geqslant 1$. Следующие утверждения равносильны.

1. $X$ является борелевским множсеством класса $F_{\alpha}(Z)\left(\right.$ класса $\left.U_{\alpha}(Z)\right)$ для некоторого полного метризуемого пространства $Z$, содержащего $X$.

2. $Y$ является борелевским множеством класса $F_{\alpha}(S)\left(\right.$ класса $\left.U_{\alpha}(S)\right)$ для некоторого полного метризуемого пространства $S$, содержащего $Y$.

ДокаЗАтЕльство. Сушествует замкнутое подпространство $X^{\prime}$ такое, что $g\left(X^{\prime}\right)=Y$ и отображение $h=g \mid X^{\prime}: X^{\prime} \rightarrow Y$ совершенно (см. [39]). Если $X$ является слабо абсолютным борелевским множеством, то $X^{\prime}, Y$ являются слабо борелевскими множествами того же класса (см. [13], [49], [50]). Этим импликация $1 \Rightarrow 2$ доказана. Одновременно мы установили, что пространство $Y$ метризуемо.

Пусть $Y$ является борелевским подмножеством полного метрического пространства $(S, \rho)$. На $X$ существует метрика $d$ такая, что каждьй прообраз $g^{-1}(y), y \in Y$, полон. Можно считать, что $\rho\left(g\left(x_{1}\right), g\left(x_{2}\right)\right) \leqslant d\left(x_{1}, x_{2}\right)$ для всех $x_{1}, x_{2} \in X$. Обозначим через $(Z, d)$ пополнение Хаусдорфа метрического пространства $(X, d)$. Тогда существует непрерьвное отображение $f: Z \rightarrow S$ такое, что $g=f \mid X$. Положим $X_{1}=f^{-1}(Y)$. В силу предложения $9.1 X$ является $G_{\delta}$-множеством пространства $X_{1}$. По построению $X_{1}$ является борелевским подмножеством в $Z$ того же класса, что $Y$ в $S$. Пространства $X$ и $X_{1}$ принадлежат тем же борелевским классам пространства $Z$. Импликация $2 \Rightarrow 1$ доказана. Доказательство завершено.

9.3. ЗАмЕчАниЕ. Если отображение $g: X \rightarrow Y$ метризуемого пространства $X$ на паракомпакт $Y$ равномерно полно и $\tau$ является кардинальным числом, то $X$ является слабо абсолютным $\tau$ - $A$-множеством тогда и только тогда, когда таковым является пространство $Y$.

9.4. ЗАмечАниЕ. Аналог теоремы 9.2 справедлив для $\Psi$-классов множеств, где $\Psi$ является допустимой операцией. Для доказательства достаточно применить следствие 17.3 из [13].

9.5. ЗАмечАниЕ. Сохранение классов множеств при различных отображениях было рассмотрено в работах [3], [46], [52]-[55], [9]-[14], [16], [49], [50]. 
9.6. Пример. Каждое компактное отображение $f: X \rightarrow Y$ равномерно полно.

Следующий пример для других целей был построен Э. Майклом [40].

9.7. ПримеР. Пусть $Y$ является несчетным метризуемым компактом. В $Z=Y \times Y$ рассмотрим семейство $\left\{\Phi_{\alpha}: \alpha<c=2^{\omega}\right\}$ всех компактных подмножеств $\Phi$, для которых первая проекция $p_{1}(\Phi)$ несчетна, где $p_{1}(u, v)=u$ для всех $(u, v) \in Z$. По индукции строятся трансфинитные последовательности точек $\left\{x_{\alpha}, y_{\alpha}, u_{\alpha}, v_{\alpha}: \alpha<c\right\} \subseteq Y$, для которых:

$-\left(x_{\alpha}, u_{\alpha}\right),\left(y_{\alpha}, v_{\alpha}\right) \in \Phi_{\alpha}$ для всех $\alpha<c ;$

- $x_{\alpha} \neq y_{\alpha}$ и $x_{\alpha}, y_{\alpha} \in Y \backslash\left\{x_{\beta}, y_{\beta}: \beta<\alpha\right\}$ для каждого $\alpha<c$.

Положим $X=Z \backslash\left\{\left(x_{\alpha}, u_{\alpha}\right): \alpha<c\right\}$ и $g=p_{1} \mid X: X \rightarrow Y$. Тогда:

- $Y$ есть метризуемый компакт;

- $X$ есть пространство со счетной базой;

- $\left|(\{y\} \times Y) \backslash g^{-1}(y)\right| \leqslant 1$ для каждого $y \in Y$;

- подпространство $g^{-1}(y)$ локально компактно для любой точки $y \in Y$;

- для каждого компакта $F \subseteq X$ множество $g(F)$ счетно;

- если $y \in Y$, то $H(y)=X \cap(Y \times\{y\})$ является несчетным замкнутым подпространством пространства $X$ и каждое компактное подмножество $F \subseteq H(y)$ счетно; в частности, $H(y)$ и $X$ не являются $A$-множествами пространства $Z$;

- каждьй прообраз $g^{-1}(y)$ метризуем полной метрикой.

Таким образом, требование равномерной полноты отображения $g$ в условиях теоремы 9.2 сушественно.

9.8. ЗАмечАниЕ. Сохранение борелевских классов множеств при открытых компактных отображениях сепарабельных метризуемых пространств было изучено А. Д. Таймановьм [52].

\section{0. Проблема Хаусдорфа. Случай счетнократных отображений}

Для каждого пространства $Z$ обозначим через $B A(Z)$ совокупность всех двусторонних $A$-множеств $H$, т.е. $H \in A(L(Z))$ и $Z \backslash H \in A(L(Z))$ для всех $H \in B A(Z)$. Если $Z$ является полным сепарабельным метризуемым пространством, то $B A(Z)=B o(Z)$.

Отображение $f: X \rightarrow Y$ пространства $X$ на пространства $Y$ назьвается:

- $B A$-измеримьм, если $f^{-1}(F) \in B A(X)$ для каждого замкнутого в $Y$ множества $F$;

- $B A$-гомеоморфизмом, если отображение $f$ взаимно однозначно и $f, f^{-1}$ являются $B A$-измеримыми.

Следуюшее утверждение, усиливаюшее одну теорему П. С. Новикова и В. Я. Арсенина [3], [42], [41], было установлено в [10; теорема 6].

10.1. ТеоремА. Пусть $X$ является топологическим пространством, $Z$ является полным сепарабельным метризуемым пространством, $L \in B A(X \times Z)$, $Y=p_{X}(L)$, где $p_{X}(x, z)=x$ для всех $(x, z) \in X \times Z$, имножество $L \cap(\{x\} \times Z)$ б-компактно для всех $x \in Y$. Тогда $Y \in B A(X)$ и существует такое подмножество $H \in B A(X \times Z)$, ито $H \subseteq L, p_{X}(H)=Y$ u $g=p_{X} \mid H: H \rightarrow Y$ является ВА-гомеоморфизмом.

Из теоремы 10.1 вытекают следуюшие результаты. 
10.2. СлЕДСТВИЕ. Пусть $f: X \rightarrow Y$ является непрерывным отображением с сепарабельным ядром метризуемого пространства $X$ на метризуемое пространство $Y$ и все прообразы $f^{-1}(y), y \in Y, \sigma$-компактны. Если $X$ является ВА-множеством некоторого полного метризуемого пространства, то:

1) $Y$ является $B A$-многеством некоторого полного метризуемого пространства;

2) существует такое $B A$-мно сество $X_{1} \in B A(X)$, что $g=f \mid X_{1}: X \rightarrow Y$ является $B A$-гомеоморфизмом с сепарабельным ядром.

10.3. СлЕДСТВИЕ. Пусть $f: X \rightarrow Y$ является открытым непрерывным отображсением метризуемого пространства $X$ на метризуемое пространство $Y$, $X$ является $B A$-множеством некоторого полного метризуемого пространства и для каждой точки $y \in Y$ подпространство $f^{-1}(y)$ б-компактно (или счетно). Тогда:

1) $Y$ является $B$ А-множеством некоторого полного метризуемого пространства;

2) существует такое $B A$-множество $X_{1} \in B A(X)$, что $g=f \mid X_{1}: X_{1} \rightarrow Y$ является $B A$-гомеоморфизмом с сепарабельным ядром.

10.4. ПримеР. Пусть $X_{1}=\left\{x_{\alpha}: \alpha \in \omega_{1}\right\}$ есть несчетное дискретное пространство и $X=X_{1} \times[0,1]$. Для каждого $\alpha<\omega_{1}$ в $\{\alpha\} \times[0,1]$ фиксируем борелевское подмножество $Y_{\alpha}$ класса $\geqslant \alpha$. Пусть $Y=\bigcup\left\{Y_{\alpha}: \alpha<\omega_{1}\right\}$. Тогда $Y \in B A(X)$ и $Y$ не является борелевским подмножеством какого-либо полного метризуемого пространства. Сушествуют сепарабельное метризуемое пространство $Z$ и непрерьвное отображение $g: X \rightarrow Z$ такие, что $g^{-1}(g(Y))=Y$ и $g(Y) \in B A(Z)$. Тогда $g(Y)$ не является борелевским подмножеством пространства $Z$. В силу теоремы 8.4 существуют слабо абсолютное метризуемое $G_{\delta \sigma}$-множество $S$ и непрерывное открытое счетнократное отображение $\phi: S \rightarrow Y$ на $Y$.

\section{1. Проблема Хаусдорфа. Случай изолированных отображений}

Отображение $f: X \rightarrow Y$ пространства $X$ на $Y$ изолировано, если для каждой точки $y \in Y$ подпространство $f^{-1}(y)$ содержит изолированную точку.

11.1. ТеоремА. Пусть $f: X \rightarrow Y$ является открытым непрерывным изолированным отображением метризуемого пространства $X$ на метризуемое пространство $Y$. Тогда существует последовательность $\left\{X_{n}: n \in \mathbb{N}\right\}$ замкнутых в $X$ множеств, для которых $Y=\bigcup\left\{f\left(X_{n}\right): n \in \mathbb{N}\right\}$, для каждого $n \in \mathbb{N}$ множество $Y_{n}=f\left(X_{n}\right)$ замкнуто в $Y$ u $f_{n}=f \mid X_{n}: X_{n} \rightarrow Y_{n}$ является гомеоморфизмом.

ДокАЗАТЕльство. Существует последовательность открытых дискретных систем $\left\{\gamma_{n}=\left\{V_{\alpha}: \alpha \in A_{n}\right\}: n \in \mathbb{N}\right\}$, для которых $\bigcup\left\{\gamma_{n}: n \in \mathbb{N}\right\}$ образует базу пространства $X$ и каждая система $f\left(\gamma_{n}\right)=\left\{f\left(V_{\alpha}\right): \alpha \in A_{n}\right\}$ дискретна в $Y$.

Фиксируем $m \in \mathbb{N}$. Положим $V_{m}=\bigcup\left\{V_{\alpha}: \alpha \in A_{n}\right\}, H_{m}=\{y \in Y:$ множество $V_{m} \cap f^{-1}(y)$ одноточечно $\}$ и $X_{m}^{\prime}=V_{m} \cap f^{-1}\left(H_{m}\right)$. Множество $X_{m}^{\prime}$ замкнуто в $V_{m}$, множество $H_{m}$ замкнуто в $f\left(V_{m}\right)$ и $f \mid X_{m}^{\prime}: X_{m}^{\prime} \rightarrow H_{m}$ является гомеоморфизмом. Поскольку $H_{m}$ является $F_{\sigma}$-множеством пространства $Y$, то доказательство завершено. 
11.2. СЛЕДСТВИЕ. Пусть $f: X \rightarrow Y$ является открытым непрерывным изолированным s-отображением метризуемого пространства $X$ на метризуемое пространство $Y$. Тогда:

1) $\operatorname{dim} Y \leqslant \operatorname{dim} X$

2) если $X$ является борелевским подмножеством некоторого полного метризуемого пространства, то $Y$ является борелевским подмножеством того же класса некоторого полного метризуемого пространства;

3) если операция $\Psi$ допустима и $X$ является $\Psi$-множсеством некоторого класса некоторого полного метризуемого пространства, то и $Y$ является таковим.м.

11.3. ЗАмечАнИЕ. Условие быть $s$-отображением сушественно. Р. Поль [47] и Х. Ж.К. Юннила [22] установили, что каждое метризуемое пространство является открытым непрерывным отображением $\sigma$-дискретного метризуемого пространства при отображении с дискретными прообразами точек. Такие отображения изолированы.

11.4. ЗАмЕчАнИЕ. В случае пространств со счетной базой теорема 11.1 и следствие 11.2 установлены А. Д. Таймановым [52], [55].

\section{2. О строении подмножеств в некоторых пространствах}

В этом разделе все пространства предполагаются метризуемыми и сепарабельными, а операции - $\aleph_{0}-t s$-операциями.

Скажем, что в пространстве $X$ задана классификация подмножеств, если определена трансфинитная последовательность семейств подмножеств $\left\{\mathscr{L}_{\alpha}: 0 \leqslant \alpha<\omega_{1}\right\}$, удовлетворяюшая условиям:

1) $\mathscr{L}_{0}$ состоит из всех замкнутых подмножеств пространства $X$;

2) $\mathscr{L}_{\alpha} \subseteq \mathscr{L}_{\beta}$, как только $\alpha \leqslant \beta<\omega_{1}$

3) если $\beta<\omega_{1}$ и $H_{1}, H_{2} \in \mathscr{L}_{\beta}$, то $H_{1} \cap H_{2} \in \mathscr{L}_{\beta}$;

4) если $\alpha \geqslant 1, H \in \mathscr{L}_{\alpha}$ и $U$ открыто в $X$, то $U \cap H \in \mathscr{L}_{\alpha}$.

Каждая допустимая операция над множествами порождает определенную классификацию подмножеств.

Предположим, что фиксирована классификация подмножеств $\mathscr{L}=\left\{\mathscr{L}_{\alpha}: \alpha<\omega_{1}\right\}$.

Скажем, что подмножество $H \subseteq X$ является приводимым подмножеством класса $\alpha$, если для каждого замкнутого подмножества $Y$ пространства $X$ из $Y \cap H \neq \varnothing$ следует, что сушествует открытое подмножество $U$ пространства $X$ такое, что $U \cap Y \cap H \neq \varnothing$ и $U \cap H \cap Y \in \bigcup\left\{\mathscr{L}_{\beta}: \beta<\alpha\right\}$.

Для каждого $\alpha<\omega_{1}$ и $H \in \mathscr{L}_{\alpha}$ однозначно определяются два подмножества $r(H)$ и $i r(H)$, удовлетворяющие условиям: $r(H) \cap i r(H)=\varnothing ; r(H) \cup i r(H)=H$; если $U$ открыто в $X$ и $U \cap \operatorname{ir}(H) \neq \varnothing$, то $U \cap \operatorname{ir}(H) \in \mathscr{L}_{\alpha} \backslash \bigcup\left\{\mathscr{L}_{\beta}: \beta<\alpha\right\} ;$ множество $r(H)$ приводимо класса $\alpha$. Будем назьвать $r(H)$ приводимой частью, а $\operatorname{ir}(H)$ - неприводимой частью множества $H$. Если $H=i r(H)$, то будем говорить, что $H$ является неприводимым множеством $\mathscr{L}$-класса $\alpha$.

Множество $H \subseteq X$ назьвается универсальньм множеством $\mathscr{L}$-класса $\alpha$, если $H \in \mathscr{L}_{\alpha}$ и для каждого $P \in \mathscr{L}_{\alpha}$ сушествует замкнутое в $X$ множество $Y$ такое, что $P$ и $H \cap Y$ гомеоморфны. 
12.1. ОПРЕДЕЛЕНИЕ. Пространство $X$ назьвается $B$-устойчивым, если оно бесконечно, имеет счетную базу и существует замкнутое подмножество $F \subseteq X$, гомеоморфное пространству $X^{\mathbb{N}}$.

12.2. ОПРЕДЕЛЕНИЕ. Пространство $X \quad B$-однородно, если оно $B$-устойчиво, топологически однородно и семейство $H(X)=\{U \subseteq X: U$ открыто и гомеоморфно пространству $X\}$ образует базу пространства $X$.

12.3. ПримеРЫ. 1. Если $Y$ является неодноточечньм пространством со счетной базой, то $X=Y^{\mathbb{N}} B$-устойчиво.

2. Пусть $X$ есть дискретная сумма пространства $\mathbb{N}^{\mathbb{N}}$ и некоторого счетного пространства. Тогда пространство $X \quad B$-устойчиво и не $B$-однородно.

3. Пусть $H(Y)$ образует базу неодноточечного однородного пространства $Y$. Тогда пространство $Y^{\mathbb{N}} B$-однородно.

4. Пространство иррациональных чисел $B(\omega)=\mathbb{N}^{\mathbb{N}} B$-однородно.

5 . Канторово совершенное множество $B(2) B$-однородно.

6. Любое сепарабельное бесконечномерное банахово пространство $B$-однородно.

7. Евклидово пространство $E^{n}$ не $B$-устойчиво.

12.4. ТеОремА. Пусть пространство X В-устойчиво. Тогда для любой операчии $\Psi$ в $X$ существует $\Psi$-универсальное множество, т.е. такое множество $L \in \Psi(X)$, что для каждого $P \in \Psi(X)$ существует замкнутое в $X$ множество $F$ такое, что $P$ и $L \cap F$ гомеоморфныл.

ДокаЗАтельство. Рассмотрим проекцию $\pi: X \times X \rightarrow X$, где $\pi(x, y)=x$ для всех $x, y \in X$. Тогда в $X \times X$ сушествует $\Psi$-универсальное множество, т.е. такое множество $H \in \Psi(X \times X)$, что для каждого $P \in \Psi(X)$ сушествует точка $y \in X$ такая, что $P=\pi(H \cap(X \times\{y\}))$ (см. [13; теорема 8.5]). Сушествуют замкнутое подмножество $\Phi \subseteq X$ и гомеоморфизм $\phi: X \times X \rightarrow \Phi$. Множество $L=\phi(H) \Psi$-универсально в $X$. Доказательство завершено.

12.5. Теорема. Пусть пространство $X$ В-устойчиво. Если $\alpha \geqslant 0, \mathscr{L}_{\alpha} \backslash$ $\bigcup\left\{\mathscr{L}_{\beta}: \beta<\alpha\right\} \neq \varnothing$ и $\mathscr{L}_{\alpha}=\Psi(X)$ для некоторой $\delta$ s-операчии $\Psi$, то в $X$ существует некоторое $\Psi$-универсальное неприводимое множество $\mathscr{L}$-класса $\alpha$.

ДокАЗАТЕльство. В силу теоремы 12.4 в $X$ существует некоторое $\Psi$-универсальное множество $H$. Существуют замкнутое подмножество $\Phi \subseteq X$ и гомеоморфизм $\psi: X^{\mathbb{N}} \rightarrow \Phi$. Множество $H_{1}=\psi\left(H^{\mathbb{N}}\right)$ искомое. Доказательство завершено.

12.6. СлЕДСтВИЕ. Пусть пространство $X$ B-устойчиво и $\delta$-операчия $\Psi$ допустима. Тогда для кажсдого $\alpha<\omega_{1}$ в $X$ существует неприводимое $\Psi$-универсальное множество $H \in \Psi_{\alpha}(X)$ класса $\alpha$. Более того, $\Psi_{\alpha}(X) \backslash \bigcup\left\{\Psi_{\beta}(X)\right.$ :

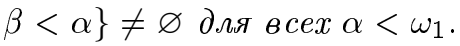

Множество $H \in \mathscr{L}_{\alpha}$ назьвается каноническим элементом $\mathscr{L}$-класса $\alpha$, если $H$ удовлетворяет условиям:

- $H$ является пространством первой категории;

- $H$ является универсальным множеством $\mathscr{L}$-класса $\alpha$;

- $H$ неприводимо $\mathscr{L}$-класса $\alpha$;

- пространство $H$ однородно, т.е. для любых точек $a, b \in H$ существует гомеоморфизм $\phi: H \rightarrow H$ такой, что $\phi(a)=b$. 
12.7. ПРЕДЛОЖЕНИЕ. Пусть пространство $X$ В-устойчиво, множество $\Phi$ замкнуто в $X$, множество $Q \subseteq X$ гомеоморфно пространству рациональных чисел, $\varphi: X \times X \rightarrow \Phi$ является гомеоморфизмом, $\Psi$ является $\delta$-операцией, $1 \leqslant \alpha<\omega_{1}$, a $H_{1} \in \Psi_{\alpha}(X)$ u $H=\phi\left(H_{1} \times Q\right)$. Тогда:

1) $Н$ является пространством первой категории;

2) если $H_{1} \in \Psi_{\alpha}(X)$ и $H_{1}$ универсально для $\Psi$-класса $\alpha$, то $Н$ универсально для $\Psi$-класса $\alpha$

3) если $H_{1}$ однородно, то и Н однородно;

4) если $H \in \Psi_{\alpha}(X)$ и $H_{1}$ неприводимо класса $\alpha$, то и $H$ неприводимо $\Psi$-класca $\alpha$

5) если $H \in \Psi_{\alpha}(X)$ и $H_{1}$ однородно и неприводимо $\Psi$-класса $\alpha$, то $H$ является каноническим множеством $\Psi$-класса $\alpha$;

6) если $\Psi \geqslant \sigma$, то $H \in \Psi_{\alpha}(X)$;

7) если $\alpha \geqslant 2$ и операция $\Psi$ допустима, то $H \in \Psi_{\alpha}(X)$.

ДокАЗАТЕЛЬСТвО проводится стандартным образом.

Для заданной классификации множеств $\mathscr{L}=\left\{\mathscr{L}_{\alpha}: \alpha<\omega_{1}\right\}$ в заданном пространстве $X$ возникают следующие проблемы.

ПроБлемА 1 (проблема непустоты классов). При каких условиях $\mathscr{L}_{\alpha} \backslash \bigcup\left\{\mathscr{L}_{\beta}\right.$ : $\beta<\alpha\} \neq \varnothing$ для всех $2 \leqslant \alpha<\omega_{1}$ ?

ПроБлемА 2 (проблема существования). Построить для каждого $\alpha<\omega_{1}$ эффективно (т.е. без привлечения аксиомы выбора):

a) определенньй элемент класса $\alpha$, т.е. элемент из $L_{\alpha} \backslash \bigcup\left\{L_{\beta}: \beta<\alpha\right\}$;

b) неприводимьй элемент класса $\alpha$;

c) канонический элемент класса $\alpha$.

ПроБЛЕмА 3. Осуществить классификацию неприводимых элементов заданного класса. При этом два множества эквивалентны, если они гомеоморфны.

ПроБлемА 4. Осушествить классификацию канонических элементов класса $\alpha$.

Проблема 5 (проблема нетривиальности). При каких условиях $\bigcup\left\{\mathscr{L}_{\alpha}: \alpha<\omega_{1}\right\}$ не совпадает с семейством $2^{X}$ всех подмножеств множества $X$.

ПроБлемА 6 (проблема эффективной нетривиальности). Построить эффективно подмножество $H \notin \bigcup\left\{\mathscr{L}_{\alpha}: \alpha<\omega_{1}\right\}$.

Проблема 5 решается тривиально, если $\bigcup\left\{\mathscr{L}_{\alpha}: \alpha<\omega_{1}\right\}=r(\Psi, X)$ для некоторой операции $\Psi$ и пространство $X$ несчетно. В этом случае каждый класс $\mathscr{L}_{\alpha}$, а значит и $r(\Psi, X)$, содержит не более континуума элементов, а семейство всех подмножеств $2^{X}$ имеет мошность больше континуума. Следовательно, $2^{X} \backslash r(\Psi, X) \neq \varnothing$.

Проблема 1 для $B$-устойчивых пространств и допустимых операций $\Psi$ решается или методом универсальных множеств, или методом А.Н. Колмогорова (см. [13], [37], $[36])$.

Проблемы 2-4 возникли для различных классификаций борелевских множеств пространства $B(\omega)$. Семейства $\left\{\mathscr{F}_{\alpha}(X): \alpha<\omega_{1}\right\}$ и $\left\{\mathscr{U}_{\alpha}(X): \alpha<\omega_{1}\right\}$ составляют классификацию Бореля. Если $\sum_{\alpha}(X)=\mathscr{F}_{\alpha}(X)$ и $\prod_{\alpha}(X)=\mathscr{U}_{\alpha}(X)$ для нечетных $\alpha$, a $\sum_{\alpha}(X)=\mathscr{U}_{\alpha}(X)$ и $\prod_{\alpha}(X)=\mathscr{F}_{\alpha}(X)$ для четных $\alpha$, то получим классификацию Хаусдорфа борелевских множеств. 
Пусть Limi $A_{n}=\bigcup\left\{\bigcap\left\{A_{n+m}: m \in \mathbb{N}\right\}: n \in \mathbb{N}\right\}$ и Lims $A_{n}=\bigcap\left\{\bigcup\left\{A_{n+m}:\right.\right.$ $m \in \mathbb{N}\}: n \in \mathbb{N}\}$ - это нижний и верхний пределы последовательности множеств $\left\{A_{n}: n \in \mathbb{N}\right\}$. Ясно, что Limi и Lims являются $\delta s$-операциями. Эти операции сильнее операций $\delta$ и $\sigma$, но они не нормальны. Если $\operatorname{Limi} A_{n}=\operatorname{Lims} A_{n}$, то последовательность $\left\{A_{n}: n \in \mathbb{N}\right\}$ назьвается сходящейся и пишут $\operatorname{Lim} A_{n}=\operatorname{Limi} A_{n}=\operatorname{Lims} A_{n}$. Если $K_{0}$ состоит из всех открыто-замкнутых подмножеств $B(\omega)$, а $K_{\alpha}$ состоит из пределов $\operatorname{Lim} A_{n}$ множеств из $\bigcup\left\{K_{\beta}: \beta<\alpha\right\}$, то получим классификацию Валле-Пуссена борелевских множеств.

Проблема 3 для классификаций борелевских множеств в $B(\omega)$ была поставлена П. С. Александровым и П. С. Урысоном [2]. Эта проблема решена А.В. Островским [45; теорема 11].

Для классификации Валле-Пуссена проблема 2а) была поставлена Лебегом, проблема 2c) - Бэром, а проблема 4 - Н. Н. Лузиным (см. [36], [37]). При этом Н. Н. Лузиным был сформулирован и вопрос о нахождении удачного определения "канонического" борелевского множества класса $\alpha$. Эти проблемы были успешно решены в работах Л. В. Келдыш [24]-[32] (см. также [37], [36]). Л. В. Келдьш [24], [25], [30]-[32] нашла удачное определение понятия канонического элемента заданного класса, для каждого $\alpha \geqslant 4$ эффективно построила пример канонического элемента класса $K_{\alpha}$ и установила справедливость следуюших двух теорем.

TK-1. Два канонических әлемента класса $K_{\alpha}, \alpha \geqslant 2$, гомеоморфны.

TK-2. Если $\alpha \geqslant 2$ и $H \in K_{\alpha} \backslash \bigcup\left\{K_{\beta}: \beta<\alpha\right\}$, то существуют әлементы $\left\{H_{n}: n \in \mathbb{N}\right\} \subseteq \bigcup\left\{K_{\beta}: \beta<\alpha\right\}$ такие, что $\bigcup\left\{H_{n}: n \in \mathbb{N}\right\} \subseteq H$ и множество $H^{\prime}=H \backslash\left\{H_{n}: n \in \mathbb{N}\right\}$ или пусто, или является каноническим әлементом класса $\alpha$.

В связи с этими результатами Л. В. Келдьш [32] сформулировала следуюшие задачи.

Проблема 7. Пусть $\alpha \geqslant 2$ и $H \in K_{\alpha} \backslash \bigcup\left\{K_{\beta}: \beta<\alpha\right\}$. Верно ли, что $H=H_{1} \cup H_{2}$, где $H_{1}$ есть канонический элемент класса $\alpha$ и $H_{2} \in \bigcup\left\{K_{\beta}: \beta<\alpha\right\}$ ?

ПроБлема 8. Пусть $\alpha \geqslant 1$ и $H \in K_{\alpha} \backslash \bigcup\left\{K_{\beta}: \beta<\alpha\right\}$. Верно ли, что $H$ является универсальным элементом для класса $K_{\alpha}$ ?

Проблема 8 решена в [45]. В работе [45] получено новое описание канонических элементов класса $K_{\alpha}$. Проблема 7 остается открытой. Представляет интерес возможность перенесения результатов Л. В. Келдьш на более обшие пространства, например, на полные $B$-однородные пространства и, в частности, на бесконечномерные банаховые пространства.

\section{СПИСОК ЛИТЕРАТУРЫ}

[1] P.S. Alexandroff. Sur la puissance des ensembles mesurables $B$ // C. R. Acad. Sci. Paris. 1916. V. 162. P. 323-325

[2] P.S. Alexandroff, P.S. Urysohn. Über nulldimensionale Punktmengen // Math. Ann. 1927. V. 98. P. $89-106$.

[3] В.ศ. Арсенин. Природа проекций некоторых $B$-множеств // Изв. АН СССР. Сер. матем. 1940. T. 4. № 4. C. 403-410.

[4] А.В.Архангельский. Отображения и пространства // УМН. 1966. Т. 21. №4. C. $133-184$. 
[5] В.Я. Арсенин, А. А. Ляпунов. Теория $A$-множеств // УМН. 1950. Т. 5. №5. C. $45-108$.

[6] R. Baire. Sur la représentation des fonctions discontinues // Acta Math. 1905. V. 30. P. 34-47.

[7] J. Barwise (ed.). Handbook of Mathematical Logic. Amsterdam: North-Holland, 1977.

[8] А. В. Чернавский. Замечание к теореме Шнейдера о существовании в совершенно-нормальных бикомпактах $A$-множества, не являющегося $B$-множеством // Вестник Моск. ун-та. Матем. 1962. № 2. С. 20.

[9] М. М. Чобан. О бэровских множествах // Второй Тираспольский симпозиум по общей топологии и ее приложениям. Кишинев, 1969. С. 87-90.

[10] М. М. Чобан. О В-измеримых сечениях // Докл. АН СССР. 1972. Т. 207. № 1. С. 48-51.

[11] M. М. Чобан. Непрерьвные образы полных пространств // Труды ММО. 1974. Т. 30. C. $23-59$.

[12] M. M. Choban (Čban). Algebras and some questions of the theory of maps // General Topology and its Relations to Modern Analysis and Algebra: Proceedings of the Fifth Prague Topological Symposium, 1981, 1983. 86-97. (Sigma Ser. Pure Math. V. 3.)

[13] M.M. Чобан. Дескриптивная теория множеств и топология // Итоги науки и техники. Современ. проблемы математики. Фундамент. направления. М.: ВИНИТИ, 1989. C. $173-237$.

[14] M. M. Choban. The open mappings and spaces // Rend. Circ. Mat. Palermo, serie II. Suppl. 1992. V. 29. P. 51-104.

[15] Р. Экгелькинг. Общая топология. М.: Мир, 1986.

[16] R. C. Freiwald. Images of Borel sets and $k$-analytic sets // Fund. Math. 1972. V. 75. № 1. P. $35-46$.

[17] Z. Frolik. A survey of separable descriptive theory of sets and spaces // Czechoslovak Math. J. 1970. V. 20. P. 406-467.

[18] Z. Frolik. Topological methods in measure theory and the theory of measurable spaces // General Topology and its Relations to Modern Analysis and Agebra (Proceedings of the Third Prague Topological Symposium, 1971). Prague: Academia, 1972. P. 127-139.

[19] F. Hausdorff. Mengenlehre. Berlin: de Gruyter, 1927.

[20] F. Hausdorff. Über innere Abbildungen // Fund. Math. 1934. V. 23. P. 279-291.

[21] J. E. Jayne, I. Namioka, C. A. Rogers. Topological properties of Banach spaces // Proc. London Math. Soc. (3). 1993. V. 66. № 3. P. 651-672.

[22] H. J. K. Junnila. Stratifiable pre-images of topological spaces // Colloq. Math. Soc. János. Bolyai. 1980. V. 23. P. 689-703.

[23] В. Г. Кановей. Проективная иерархия Н. Н. Лузина: Современное состояние теории // Справочная книга по математической логике. Ч. II. М.: Наука, 1982. С. 273-370.

[24] Л.В. Келдыш. О гомеоморфности канонических элементов третьего класса // Матем. сб. 1934. Т. 41. № 2. С. 187-220.

[25] Л.В. Келдыш. О простых функциях класса $\alpha$ // Докл. АН СССР. 1934. Т. 4. № 4. C. $192-197$.

[26] Л. В. Келдыш. Счетные измеримые $B$-решета, определяющие множества измеримые $B$ // Изв. АН СССР. Сер. матем. 1937. № 3. С. 403-418.

[27] Л. В. Келдыш. Об одном свойстве решет измеримых $B$ // Изв. АН СССР. Сер. матем. 1938. № 1. C. 125-135.

[28] Л.В. Келдыш. Решета, определяющие множества измеримые B // Докл. АН СССР. 1938. T. 19. №1-2. С. 11-14.

[29] Л. В. Келдыш. Структура минимальных решет, определяюших множества измеримые $B$ // Изв. АН СССР. Сер. матем. 1938. № 2. С. 221-248.

[30] Л. В. Келдыш. Однородные множества измеримые В // Докл. АН СССР. 1940. Т. 26. № 6. C. 531-534.

[31] Л. В. Келдыш. Структура B-множеств // Докл. АН СССР. 1941. Т. 31. № 7. С. 651-653.

[32] Л. В. Келдыш. Структура $B$-множеств // Труды МИАН. 1945. Т. 17.

[33] Л. В. Келдыш. Об открытых отображениях $A$-множеств // Докл. АН СССР. 1945. Т. 49. № 9. С. $646-648$.

[34] К. Куратовский. Топология. Т. 1. М.: Мир, 1966. 
[35] H. Lebesgue. Sur les fonctions représentables analytiquement // J. Math. (6). 1905. V. 1. P. $139-216$.

[36] N. N. Lusin. Leçons sur les ensembles analytiques et leurs applications. Paris: Gauthier-Villars, 1930.

[37] Н. Н. Лузин. Собрание сочинений. Т. 2. Дескриптивная теория множеств. М.: Изд-во АН CCCP, 1958.

[38] А. А. Ляпунов. Вопросы теории множеств и теории функций. М.: Наука, 1979.

[39] E. Michael. A theorem on semi-continuous set-valued functions // Duke Math. J. 1959. V. 26. P. $647-651$

[40] E. Michael. $G_{\delta}$-sections and compact-covering maps // Duke Math. J. 1969. V. 36. P. $125-127$.

[41] P.S. Novikoff. Sur les fonctions implicites mesurables B // Fund. Math. 1931. V. 17. P. $8-25$.

[42] П. С. Новиков. Теория множеств. Математическая логика и алгебра. Избранные труды. M.: Наука, 1979.

[43] Ю. С. Очан. Обобщенная $A$-операция // Докл. АН СССР. 1941. Т. 33. №6. С. 393-395.

[44] Ю. С. Очан. Теория операций над множествами // УМН. 1955. Т. 10. № 3. С. 71-128.

[45] А. В. Островский. К вопросу Л.В. Келдыш о структуре борелевских множеств // Матем. сб. 1986. Т. 131. № 3. С. 323-346.

[46] Б. А. Пасынков. Об открытых отображениях // Докл. АН СССР. 1967. Т. 175. № 2. C. $292-295$.

[47] R. Pol. On category-raising and dimension-raising open mappings with discrete fibers // Colloq. Math. 1981. V. 44. № 1. P. 65-76.

[48] В.И. Пономарев. О борелевских множествах в совершенно-нормальных бикомпактах // Докл. АН СССР. 1966. Т. 170. №3. С. 520-523.

[49] C. A. Rogers, J.E. Jayne, C. Dellacherie, F. Topsoe, J. Hoffmann-Jørgensen, D. A. Martin, A.S. Kechris, A. H. Stone. Analytic Sets. London: Academic Press, 1980.

[50] J. Saint-Raymond. Fonctions boreliennes sur un quotient // Bull. Sci. Math. (2). 1976. V. 100. № 2. P. 141-147.

[51] A. H. Stone. Non-separable Borel sets // Rozprawy Math. 1962. V. 28.

[52] А. Д. Тайманов. Об открытых образах борелевских множеств // Матем. сб. 1955. Т. 37. № 2. C. 293-300.

[53] А. Д. Тайманов. О замкнутых отображениях. I // Матем. сб. 1955. Т. 36. № 2. C. $349-352$

[54] А. Д. Тайманов. О замкнутых отображениях. II // Матем. сб. 1960. Т. 52. №1. C. $579-588$

[55] А. Д. Тайманов. О некоторых работах, связанных с дескриптивной теорией множеств и топологией // Труды МИАН. 1973. Т. 83. С. 203-213.

[56] H. H. Wicke, J.M. Worrell, Jr. Open continuous images of certain kinds of $M$-spaces and completeness of mappings and spaces // General Topology and Appl. 1971. V. 1. №1. P. $85-100$.

Тирасполский государственный университет,

Поступила в редакцию

Республика Молдова

11.05 .2005

E-mail: mmchoban@mail.md 